\title{
Composição química e energia metabolizável de híbridos de milho para frangos de corte ${ }^{1}$
}

\author{
Rodrigo de Oliveira Vieira ${ }^{2}$, Paulo Borges Rodrigues ${ }^{3}$, Rilke Tadeu Fonseca de Freitas ${ }^{3}$, \\ Germano Augusto Jerônimo do Nascimento ${ }^{4}$, Edson Lindolfo da Silva ${ }^{4}$, Renato Hespanhol ${ }^{5}$
}

\footnotetext{
1 Parte da dissertação de Mestrado do primeiro autor - financiada pelo CNPq

2 Zootecnista/UNESP - Jaboticabal, MS em Nutrição Monogástricos/UFLA.

${ }^{3}$ Departamento de Zootecnia/UFLA. Bolsista do CNPq.

${ }^{4}$ Doutorando do Programa de Pós-graduação em Zootecnia/UFLA.

${ }^{5}$ Graduando em Zootecnia - Bosista PIBIC/CNPq/UFLA.
}

RESUMO - Determinaram-se a composição química e os valores energéticos de 45 híbridos de milho em quatro ensaios de metabolismo com pintos em crescimento. Foram utilizados 1.225 pintos machos com 19 dias de idade (350 nos ensaios 1, 2 e 3 e 175 no ensaio 4). Os ensaios 1, 2 e 3 foram compostos de 14 tratamentos, constituídos, cada um, de 13 dietas-teste com híbridos de milho e uma dieta-referência. O ensaio 4 foi composto de sete tratamentos, constituídos de seis dietas-teste e uma dietareferência. Em todos os ensaios, os milhos substituíram $40 \%$ da dieta-referência. Adotou-se delineamento inteiramente casualizado, com cinco repetições de 5 aves/parcela. As dietas e a água foram fornecidas à vontade durante sete dias (quatro de adaptação e três de coleta total de excretas). Os valores percentuais de PB variaram em 32\% (7,79\% vs 11,45\%, expressos na MS) e os de energia bruta (EB), em 5,2\%. O menor valor foi $4.425 \mathrm{kcal}$ e o maior, $4.668 \mathrm{kcal} / \mathrm{kg}$ de MS. O valor médio de energia metabolizável aparente corrigida (EMAn) foi de $3.744 \mathrm{kcal} / \mathrm{kg}$ e apresentou variação de 15,15\% entre os híbridos testados (3.405 a $4.013 \mathrm{kcal} / \mathrm{kg}$ ). Entretanto, os dois híbridos que apresentaram esta variação de $608 \mathrm{kcal} / \mathrm{kg}$ de MS na EMAn tiveram valores de EB semelhantes, diferentes em apenas 0,36\% (3.914 e $3.931 \mathrm{kcal}$ de EB/kg de MS), o que possivelmente resultou do coeficiente de metabolizabilidade da EB, que foi de 75\% para o híbrido de menor EMAn e de 88\% para o de maior EMAn. Apesar de ser um alimento energético, os valores protéicos e energéticos dos diferentes híbridos variaram consideravelmente.

Palavras-chave: alimento, coeficiente de metabolizabilidade, digestibilidade

\section{Chemical composition and metabolizable energy of corn hybrids for broilers}

\begin{abstract}
The values of chemical composition and metabolizable energy of 45 hybrid corns were determined in four trials with growing chickens. A total of 1,225, nineteen days old male chicks were used: 350 in the trials 1,2 and 3 and 175 in trial 4. In the trials 1,2 and 3 the treatments consisted of 13 test diets, being 13 corn varieties, and the reference diet in each trial. Trial 4 consisted of 7 treatments, being 6 test diets (corn varieties) and the reference. The hybrid corns, replaced $40 \%$ of the reference diets in all the trials.. A completely randomized design with five replicates of five birds per cage was used. The diets and water were offered ad libitum for a 7-day period, being four days for adaptation and three for days for excreta collection. A variation of 32\% in crude protein - CP (7.79\% vs. $11.45 \%$, dry matter basis) was found. The values of gross energy (GE) presented a variation of 5.2\%. The higher value observed was 4,668 kcal and the lower value 4,425 $\mathrm{kcal} / \mathrm{kg}$. The average value for corrected apparent metabolizable energy (AMEn) was 3,744 kcal/kg, with a variation of 15.15\% (3,405 to 4,013 kcal/kg). However, it was observed that the two hybrids presenting this variation (608 kcal AMEn/kg DM) had similar GE values (3914 and $3931 \mathrm{kcal} \mathrm{GE} / \mathrm{kg}$ of DM; $0.36 \%$ variation). This variation in the AMEn can possibly be accounted on the coefficient of gross energy metabolization which was $75 \%$ for the hybrid with the lowest AMEn and $88 \%$ for the hybrid with the highest AMEn. Despite of corn being an energy source, the evaluation of its CP content is important due to the considerable variation in the protein values of the different hybrids found currently. The same is valid for the energy values.
\end{abstract}

Key Words: digestibility, feed, metabolizability coefficient

\section{Introdução}

Nas últimas décadas, a agropecuária brasileira atingiu alto nível tecnológico, o que a deixou em situação privilegiada em âmbito mundial. Essa evolução, em geral, propor- cionou fatores positivos, entretanto, determinou também o surgimento de alguns impactos negativos, como a redução do lucro por unidade de produto (carne, leite e ovo), diminuindo a rentabilidade, principalmente para pequenos e médios produtores. 
Neste cenário, a avicultura foi uma das atividades que mais sofreram impacto, haja vista a pequena diferença entre a relação custo de produção $\times$ preço pago pelo frango ao produtor. Alguns fatores podem diminuir este impacto, entre eles o investimento em tecnologias apropriadas, a produção em escala e a diminuição do custo de produção.

As dietas comumente usadas na avicultura de corte, em geral, têm o milho como principal ingrediente e fonte de energia, o que torna esse alimento responsável, em média, por mais de $20 \%$ da proteína, $10 \%$ de lisina e $25 \%$ da metionina+cistina presentes nas dietas. No entanto, variações significativas são encontradas na composição química e no valor nutricional deste grão, dificultando, assim, a formulação precisa das rações. Segundo dados apresentados por Lima (2000), no período de 1979 a 1997, em virtude da variação na composição química dos milhos, ocorreram oscilações de 1,41 a 6,09\% nos teores de óleo e de 6,43 a 10,99\% nos valores de proteína. No período estudado, verificou-se que o nível de energia metabolizável aparente variou de 3.045 a $3.407 \mathrm{kcal} / \mathrm{kg}$ e o de energia metabolizável verdadeira, de 3.440 a $3.820 \mathrm{kcal} / \mathrm{kg}$ de matéria natural.

Um problema a ser considerado é que, na maioria, as dietas são formuladas com base nos valores descritos em tabelas de composição de alimentos. Contudo, a composição média do milho nas tabelas pode diferir da composição do milho utilizado e, conseqüentemente, as dietas fornecidas podem extrapolar as exigências nutricionais dos animais. Assim, tem-se buscado constantemente a formulação de dietas mais eficientes e economicamente viáveis e o aumento de pesquisas envolvendo a composição química e os valores de digestibilidade dos nutrientes do milho.

Como destacado por Santos et al. (2005), o conhecimento dos dados de composição química, dos valores de digestibilidade e da disponibilidade de nutrientes constitui a melhor forma de balanceamento de dietas técnica e economicamente viáveis.

O conhecimento da composição química e dos valores energéticos de híbridos de milho pode permitir a elaboração de equações de predição que permitam a estimativa mais próxima do valor energético do milho a partir de sua composição química. Dessa forma, o objetivo neste estudo foi determinar a composição química e os valores energéticos de 45 híbridos de milho para uso em dietas para frangos de corte.

\section{Material e Métodos}

Quatro experimentos de metabolismo foram conduzidos no período de dezembro de 2004 a maio de 2005 no Setor de Avicultura do Departamento de Zootecnia (DZO) da Universidade Federal de Lavras (UFLA), localizada em
Lavras, Minas Gerais. O município de Lavras está situado a 910 m, 24 ${ }^{\circ} 14^{\prime}$ 'de latitude Sul e $45^{\circ} 00^{\prime}$ ' de longitude Oeste.

Foram utilizados 1.225 pintos de corte machos da linhagem Cobb-500, criados em galpão de alvenaria até os 14 dias de idade. Neste período, as aves foram alimentadas com uma dieta inicial para frangos de corte composta de ingredientes básicos, milho e farelo de soja, formulada de acordo com recomendações de Rostagno et al. (2000). Após esse período, as aves foram pesadas e transferidas para uma sala de estudos de metabolismo com controle de temperatura e iluminação artificial durante 24 horas por dia. As temperaturas médias, mínima e máxima, registradas durante o período experimental foram $22,8 \pm 1,5^{\circ} \mathrm{C}$ e $25,8 \pm 2,4^{\circ} \mathrm{C}$, respectivamente.

Para determinação dos valores de energia metabolizável aparente corrigida (EMAn) dos 45 híbridos de milho, foram conduzidos quatro ensaios de metabolismo com pintos na fase inicial (14 a 21 dias), adotando-se o método tradicional de coleta total de excretas. Em cada um dos três primeiros ensaios, determinou-se a EMAn de 13 híbridos de milho e, no ensaio 4, de seis híbridos de milho.

Em cada ensaio, os híbridos de milho foram adicionados a uma dieta-referência na qual o sorgo foi a fonte energética em substituição ao milho para assegurar que os híbridos de milho avaliados não estariam sendo confundidos com a dieta-referência (Tabela 1).

Em cada um dos três primeiros ensaios, foram utilizados 350 pintos, cujos pesos foram $345 \pm 3 \mathrm{~g}, 397 \pm 3$ g e $393 \pm 4$ g, respectivamente. No ensaio 4 , foram utilizados 175 pintos, com $388 \pm 3$ g, alimentados com dietas experimentais formuladas com seis híbridos de milho e a dieta-referência. Em todos os ensaios, os híbridos de milho substituíram $40 \%$ da dieta-referência.

Os híbridos de milho utilizados nos ensaios foram provenientes do Departamento de Agricultura da UFLA, onde foram cultivados em mesma área e colhidos na mesma época. Depois de colhidos, foram selecionados manualmente, sendo utilizados neste trabalho apenas os híbridos íntegros, livres de impurezas e aqueles que não estavam ardidos. Após a seleção, os milhos foram triturados em moinho com peneira de $2 \mathrm{~mm}$ para, posteriormente, serem misturados à dieta-referência, em proporções de $40 \%$, conforme descrito anteriormente, originando as dietas-teste.

Todos os ensaios foram conduzidos em delineamento inteiramente casualizado, com cinco repetições e cinco aves por parcela. As dietas e a água foram fornecidas à vontade por um período de sete dias, quatro de adaptação (pré-experimental) e três de coleta total de excretas (Rodrigues et al., 2005). As coletas foram realizadas uma vez ao dia, com início sempre às $8 \mathrm{~h}$. 
Tabela 1 - Composições centesimal e calculada da dietareferência

Table 1 - Percentage and calculated composition of the reference diet

\begin{tabular}{|c|c|}
\hline $\begin{array}{l}\text { Ingrediente } \\
\text { Ingredient }\end{array}$ & $(\%)$ \\
\hline Sorgo & 56,646 \\
\hline Sorghum & \\
\hline Farelo de soja & 35,066 \\
\hline Soybean meal & \\
\hline $\begin{array}{l}\text { Calcário calcítico } \\
\text { Limestone }\end{array}$ & 0,989 \\
\hline Fosfato bicálcico & 1,797 \\
\hline Dicalcium phosphate & \\
\hline $\begin{array}{l}\text { Óleo vegetal } \\
\text { Vegetable oil }\end{array}$ & 4,232 \\
\hline Sal & 0,464 \\
\hline Salt & \\
\hline $\begin{array}{l}\text { DL-metionina }(99,0 \%) \\
\text { DL-methionine }\end{array}$ & 0,169 \\
\hline L-lisina HCL $(99,0 \%)$ & 0,437 \\
\hline $\begin{array}{l}\text { L-lysine } H C L \\
\text { Suplemento vitamínico }{ }^{1} \\
\text { Vitamin mix }\end{array}$ & 0,100 \\
\hline $\begin{array}{l}\text { Suplemento mineral }{ }^{2} \\
\text { Mineral mix }\end{array}$ & 0,100 \\
\hline Total & 100,000 \\
\hline Total & \\
\hline
\end{tabular}

Composição calculada

Calculated composition

\begin{tabular}{lr}
\hline $\begin{array}{l}\text { Energia metabolizável (kcal/kg) } \\
\text { Metabolizable energy }\end{array}$ & 3.000 \\
$\begin{array}{l}\text { Proteína bruta (\%) } \\
\text { Crude protein }\end{array}$ & 20,90 \\
$\begin{array}{l}\text { Metionina + cistina (\%) } \\
\text { Methionine + cystine }\end{array}$ & 0,79 \\
$\begin{array}{l}\text { Lisina (\%) } \\
\text { Lysine }\end{array}$ & 1,12 \\
$\begin{array}{l}\text { Cálcio (\%) } \\
\text { Calcium }\end{array}$ & 0,94 \\
$\begin{array}{l}\text { Fósforo disponível (\%) } \\
\text { Available phosphorus }\end{array}$ & \\
Sódio (\%) & 0,44 \\
Sodiun
\end{tabular}

Sodium

${ }^{1}$ Conteúdo por $\mathrm{kg}$ de dieta (Content per $\mathrm{kg}$ of diet): vit. $\mathrm{A}=12.000 \mathrm{UI}$; vit. $\mathrm{D} 3=2.200 \mathrm{Ul}$; vit. $\mathrm{E}=30 \mathrm{mg}$; vit. $\mathrm{K} 3=2,5 \mathrm{mg}$; vit. $\mathrm{B} 1=2,2 \mathrm{mg}$; vit. B2 = $6 \mathrm{mg}$; vit. $\mathrm{B} 6=3,3 \mathrm{mg}$; vit. B12 $=60 \mathrm{mcg}$; ac. nicotínico (nicotinic acid $)=53 \mathrm{mg}$; ác. pantotênico $($ pantothenic acid $)=13 \mathrm{mg}$; biotina $($ biotin $)=$ $0,11 \mathrm{mg}$; ac. fólico (folic acid) $=1 \mathrm{mg}$; selênio $($ selenium $)=0,25 \mathrm{mg}$.

${ }^{2}$ Conteúdo por $\mathrm{kg}$ de dieta (Content per $\mathrm{kg}$ of diet): $\mathrm{Mn}=75 \mathrm{mg} ; \mathrm{Zn}=70 \mathrm{mg}$; $\mathrm{Cu}=8,5 \mathrm{mg} ; \mathrm{Fe}=50 \mathrm{mg} ; \mathrm{I}=1,5 \mathrm{mg} ; \mathrm{Co}=0,2 \mathrm{mg}$.

No período de coleta (19 a 21 dias de idade), as bandejas foram revestidas com plástico sob o piso de cada gaiola, a fim de evitar perdas. O consumo em cada unidade experimental durante o período de coleta foi registrado e as excretas colocadas em sacos plásticos, sendo devidamente identificadas e armazenadas em freezer até o final do período de coleta. Posteriormente, as amostras foram pesadas e homogeneizadas, sendo retiradas alíquotas para as análises laboratoriais.

Os valores de EMA foram determinados pela equação de Matterson et al. (1965) e ajustados quanto à retenção de nitrogênio. As fórmulas utilizadas foram:
EMAn da DT ou DR = EB ingerida $-($ EB excretada $+8,22 * \mathrm{BN})$

MS ingerida

em que: $\mathrm{RT}$ = dieta-teste; e $\mathrm{DR}=$ dieta-referência; $\mathrm{BN}=$ balanço de nitrogênio $=\mathrm{N}$ ingerido $-\mathrm{N}$ excretado

EMAn do híbrido de milho $=\mathrm{EMAn}_{\mathrm{DR}}+\mathrm{EMAn}_{\mathrm{DT}}-\mathrm{EMAn}_{\mathrm{DR}}$ g/g de substituição

Para cada híbrido, foram determinados os teores de MS, N, PB, EE, EB, FB, FDA, FDN, cinzas, Ca e P, conforme descrito por Silva (2002). Utilizando-se a técnica descrita por Zanotto \& Bellaver (1996), realizou-se também, para cada híbrido de milho, a determinação do diâmetro geométrico médio (DGM) dos híbridos de milho. Foram também realizadas análises de MS, N e EB das dietas experimentais e das excretas.

Utilizando os dados de EMAn e EB, expressos na matéria natural, calculou-se o coeficiente de metabolizabilidade da EB (CMEB) dos híbridos de milho, pela seguinte fórmula:

$$
\mathrm{CMEB}=(\mathrm{EMAn} / \mathrm{EB}) \times 100
$$

Todas as análises foram realizadas no Laboratório de Pesquisa Animal do DZO da UFLA.

\section{Resultados e Discussão}

A composição química e o diâmetro geométrico médio (DGM) dos 45 híbridos de milho avaliados encontram-se na Tabela 2. A composição química e os valores energéticos dos híbridos de milho apresentaram valores médios próximos aos citados por EMBRAPA (1991), NRC (1994), Lima (2001) e Rostagno et al. (2005).

Entre os valores de PB determinados, houve variação de $32 \%$ entre o menor e o maior valor (7,79 a 11,45\% na MS). Nagata et al. (2004) encontraram variação de 35,79\% (7,05 a 10,98\%) e Rodrigues et al. (2003), de 27,56\% (8,36 a 11,54\%). Para os valores de EB observados, essa variação foi de 5,2\% (4.425 a $4.668 \mathrm{kcal} / \mathrm{kg}$ ), enquanto Rodrigues et al. (2003) encontraram variação de 3,07\% (4.544 a $4.688 \mathrm{kcal} / \mathrm{kg}$ ). As variações para os outros nutrientes foram de 24,7; 66; 65,2; 44,3; 53,6; 71,1 e 36,9\%, respectivamente, para EE, FB, FDA, FDN, cinzas, cálcio e fósforo.

Possivelmente, o fator que mais influenciou os valores nutricionais foi a variedade e, no caso da proteína, segundo Vasconcellos (1989), a adubação nitrogenada. Esse último fator deve ser considerado relevante, pois híbridos cultivados em mesmas condições, porém, com diferentes doses de adubação nitrogenada, podem influenciar a com- 
Tabela 2 - Composição química e diâmetro geométrico médio (DGM) de híbridos de milho ${ }^{1}$ Table 2 - Chemical composition and medium geometric diameter (MGD) of hybrid corns

Composição $0^{2,3}$

Composition

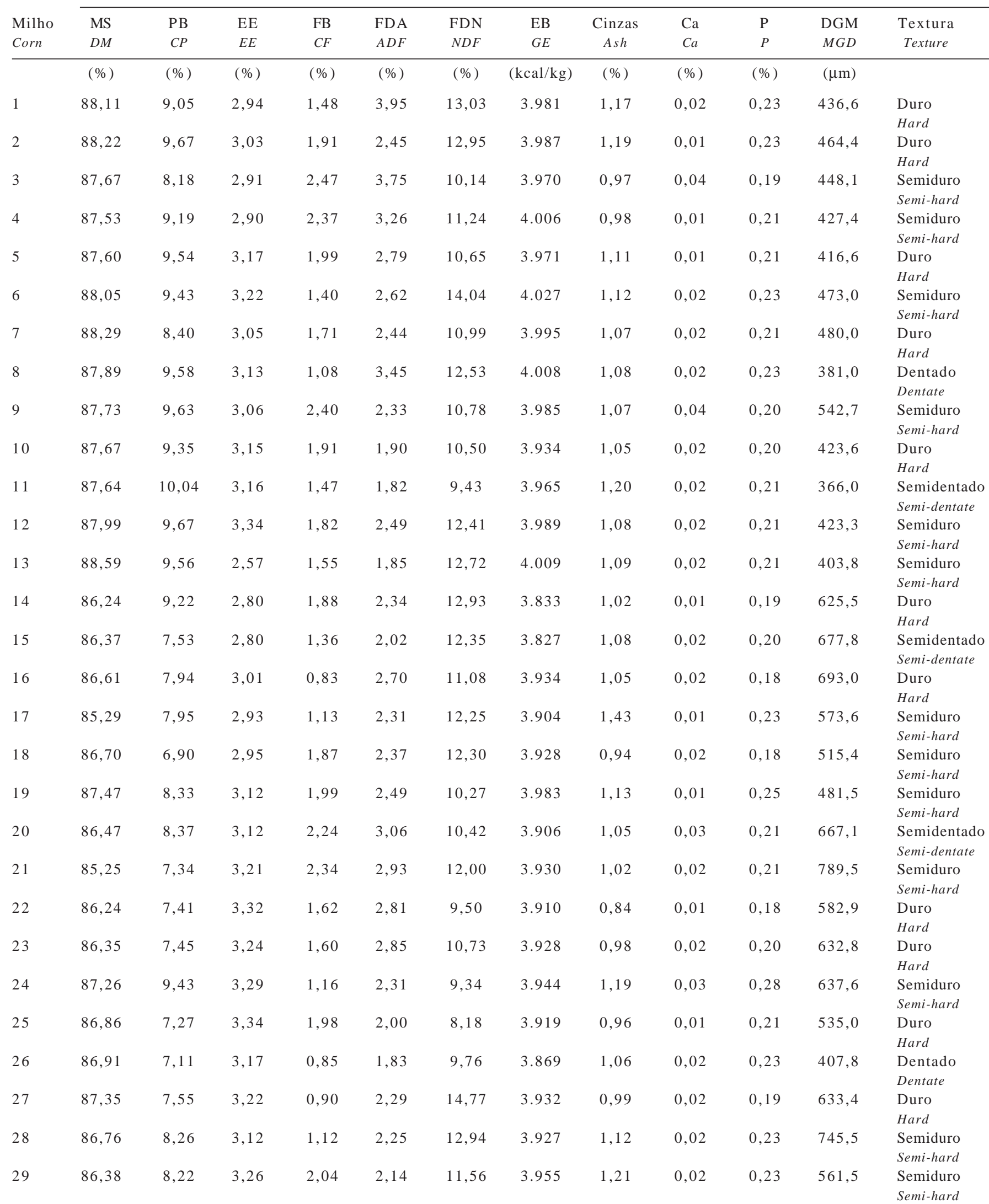


Continuação da Tabela 2 ..

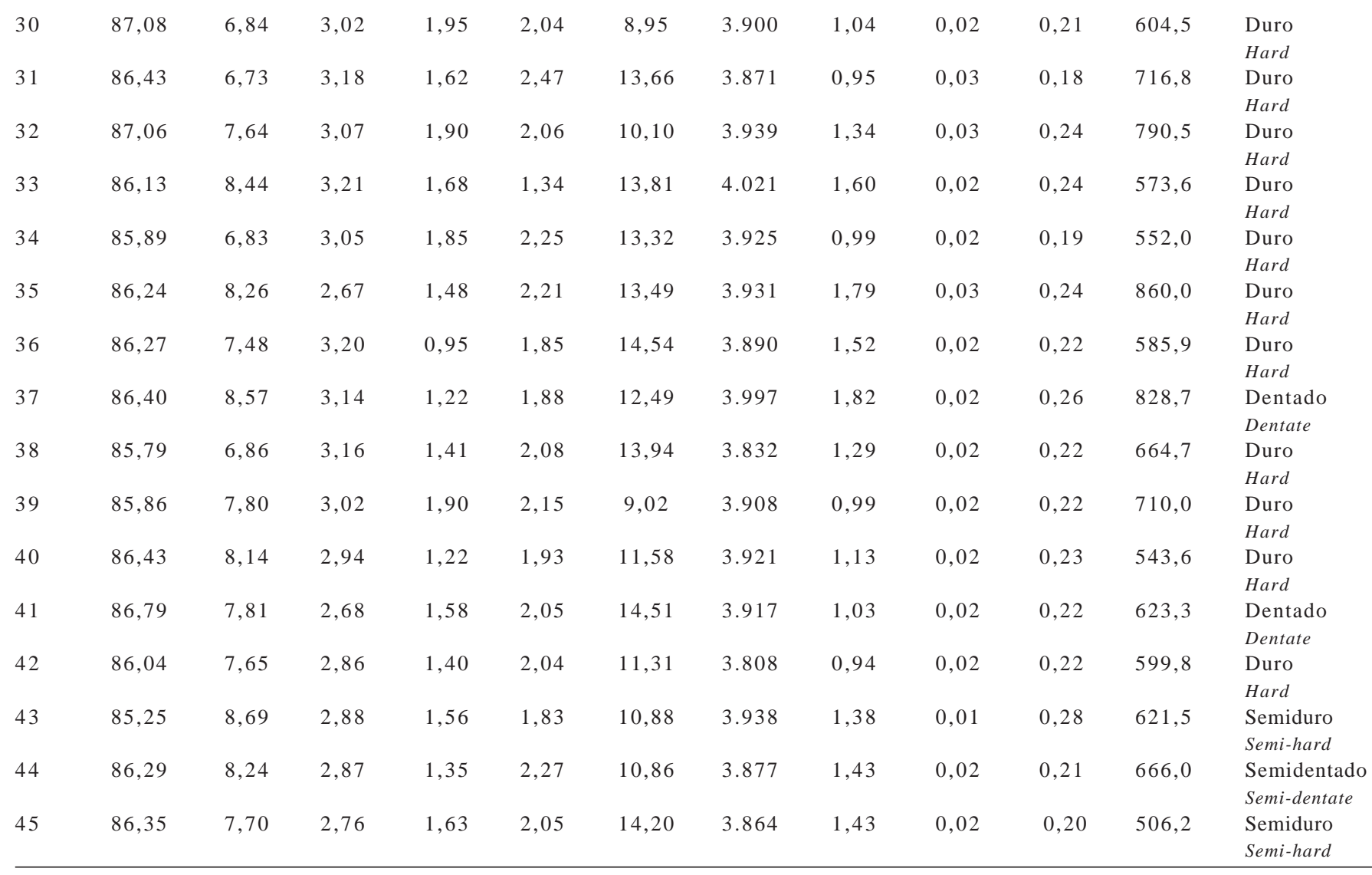

1 Valores expressos na matéria natural.

2 Análises realizadas no Laboratório de Nutrição Animal do DZO da Universidade Federal de Lavras (UFLA)

1 Values expressed on as-fed basis.

2 Analyses performed at the Animal Nutrition Laboratory of Federal University of Lavras (UFLA).

posição em PB da dieta. Os valores médios de EB e, principalmente, $\mathrm{PB}$ estão de acordo com os descritos por Rostagno et al. (2005).

Ainda quanto à variação na composição de um alimento, Bath et al. (1999) afirmaram que os valores encontrados em tabelas devem ser utilizados como orientação, e não como informação precisa, na obtenção da composição do alimento.

Os valores de diâmetro geométrico médio (DGM) encontrados foram, em média, de 575,4 $\mu \mathrm{m}$, com variação de $57,4 \%$ entre o menor e o maior valor determinado (366,0 a $860,0 \mu \mathrm{m}$ ). Segundo Nunes et al. (2001), os alimentos podem ser classificados quanto ao DGM como grossos (maiores que 832,7 $\mu \mathrm{m}$ ), médios (375,3 a 832,7 $\mu \mathrm{m}$ ) e finos (menores que $375,3 \mu \mathrm{m})$. Nessas condições, apenas o híbrido 35 apresentou DGM grosso e o restante dos híbridos pode ser classificado como de DGM médio.

As médias, os erros-padrão, os coeficientes de variação, a amplitude da composição química, do diâmetro geométrico médio e dos valores energéticos e os coeficientes de metabolizabilidade da EB dos híbridos de milho encontram-se na Tabela 3. Os menores coeficientes de variação foram encontrados para MS e EB (0,99 e 1,37, respectivamente) e os maiores, para FB e cálcio (26,05 e 35,35, respectivamente).

Considerando os resultados obtidos neste trabalho, pode-se afirmar que o DGM dos milhos não é facilmente controlado, pois esse controle depende de suas caracterís-

Tabela 3 - Valores médios, erro-padrão (EP), coeficiente de variação (CV), amplitude da composição química, do diâmetro geométrico médio e dos valores energéticos e coeficiente de metabolizabilidade da energia bruta de híbridos de milho

Table 3 - Mean, standard error (SE), coefficient of variation and range of the chemical composition, medium geometric diameter, energy values and metabolization coefficient of gross energy of hybrid corns

\begin{tabular}{|c|c|c|c|c|c|c|c|c|c|c|c|c|c|}
\hline & $\begin{array}{l}\text { MS } \\
D M\end{array}$ & $\begin{array}{l}\mathrm{PB} \\
C P\end{array}$ & $\begin{array}{l}\mathrm{EE} \\
E E\end{array}$ & $\begin{array}{l}\text { FB } \\
C F\end{array}$ & $\begin{array}{l}\text { FDA } \\
A D F\end{array}$ & $\begin{array}{l}\text { FDN } \\
N D F\end{array}$ & $\begin{array}{l}\text { EB } \\
G E\end{array}$ & $\begin{array}{c}\text { Cinzas } \\
\text { Ash }\end{array}$ & $\mathrm{Ca}$ & $\mathrm{P}$ & $\begin{array}{l}\text { DGM } \\
M G D\end{array}$ & $\begin{array}{c}\text { EMAn } \\
\text { AMEn }\end{array}$ & $\begin{array}{c}\text { CMEB } \\
\text { CGEM }\end{array}$ \\
\hline & 8 & 25 & & & 2,36 & 74 & 3. & 1 , & & & 41 & 33 & 32,66 \\
\hline $\mathrm{P}(S E)$ & 0,13 & 0,14 & 0,28 & 0,63 & 0,08 & 0,25 & 8,06 & 0,03 & 0,001 & 0,003 & 18,72 & 15,47 & 0,35 \\
\hline CV (\%) & 0,99 & 11,39 & 6,12 & 26,05 & 22,25 & 14,56 & 1,37 & 18,99 & 35,355 & 10,869 & 21,82 & 3,21 & 3,19 \\
\hline Amplitude (Range) & 3,34 & 3,31 & 0,77 & 1,64 & 2,61 & 6,59 & 219,00 & 0,98 & 0,030 & 0,100 & 494,00 & 506,00 & 13,00 \\
\hline
\end{tabular}


ticas físicas e de seu fluxo para o moinho, independentemente do diâmetro da peneira usada. Na moagem, observou-se que os híbridos mais duros e com maior taxa de passagem no moinho apresentaram maior DGM.

Os valores energéticos determinados, os desvios-padrão e os coeficientes de metabolizabilidade da energia bruta (CMEB) são descritos na Tabela 4.

O valor médio de EMAn determinado nos ensaios foi de $3.744 \mathrm{kcal} / \mathrm{kg}$ de MS, menor que os valores apresentados por Rostagno et al. (2005) e Kato (2005), de 3.881 e $3.794 \mathrm{kcal} / \mathrm{kg}$ de MS, respectivamente. Possivelmente, essa diferença está associada ao ganho de peso das aves e, conseqüentemente, ao diferente balanço de nitrogênio, cujos valores estão diretamente relacionados ao cálculo da EMAn. Os valores de EMAn foram similares aos encontrados por Nagata et al. (2004) e NRC (1994), de 3.768 e $3.764 \mathrm{kcal} / \mathrm{kg}$ de MS (menos de 1\% de variação), respectivamente, e superiores aos encontrados por Rodrigues et al. (2001) e Lima (2001), de 3.625 e 3.682 kcal/kg de MS.

A variação da EMAn entre os híbridos estudados no presente trabalho foi de 15,15\% (3.405 a $4.013 \mathrm{kcal} / \mathrm{kg}$, respectivamente, para os híbridos 41 e 35), superior aos percentuais de 3,27 e 10,63\% encontrados por Nagata et al.(2004) e Lima (2001), respectivamente. Entretanto, entre os dois híbridos citados, que apresentaram esta variação na EMAn (de $608 \mathrm{kcal} / \mathrm{kg}$ de MS), os valores de EB foram semelhantes e variaram somente 0,36\% (3.914 e 3.931 kcal de EB/kg de MS). Essa variação na EMAn, possivelmente, foi ocasionada pelo CMEB, de $75 \%$ para o híbrido 41 e de $88 \%$ para o híbrido 35.

O CMEB representa o quanto da EB do alimento é metabolizado pelo animal e, possivelmente, está relacionado às características físicas dos híbridos de milho. Os híbridos que apresentaram maior CMEB (acima de 85\%) eram duros e possuíam valores protéicos inferiores à média (9,49\%). O restante era semiduro, semidentado ou dentado. No entanto, alguns híbridos duros apresentaram CMEB inferior a 85\%.

Na Tabela 5 constam os resultados das correlações de Pearson. Analisando as variáveis em conjunto, observou-se que o EE se associou significativamente $(\mathrm{P}<0,05)$ ao valor médio do CMEB e apresentou correlação positiva e baixa (27,9\%). Do mesmo modo, o diâmetro geométrico médio das partículas apresentou correlação positiva de aproximadamente $22,1 \%$ com o CMEB médio ( $\mathrm{P}=0,0726)$, mostrando, portanto, influência da granulometria do alimento sobre o coeficiente de metabolização.

Nesse contexto, um importante recurso para análise da energia com base na composição química, e mesmo física dos alimentos, seria a disponibilidade de equações de predição, pois essas equações podem também aumentar a
Tabela 4 - Valores de energia metabolizável aparente corrigida (EMAn) e coeficiente de metabolizabilidade da enegia bruta (CMEB) de híbridos de milho determinados em frangos de corte no período de 14 a 21 dias de idade. Valores expressos na MS e na matéria natural

Table 4 - $\quad$ Apparent metabolizable energy corrected for nitrogen balance (AMEn) and metabolization coefficient of gross energy (MCGE) of hybrid corns, determinated with broiler chicks (14 to 21 days of age), in DM and as fed basis

\begin{tabular}{lccc}
\hline Milho & EMAn (MS) & EMAn (MN) & CMEB \\
Corn & AMEn (DM) & AMEn & CGEM
\end{tabular}

\begin{tabular}{llll} 
& $(\mathrm{kcal} / \mathrm{kg})$ & $(\mathrm{kcal} / \mathrm{kg})$ & $\%$ \\
\hline 1 & $3.626 \pm 76$ & $3.194 \pm 67$ & 80 \\
2 & $3.604 \pm 134$ & $3.180 \pm 118$ & 80
\end{tabular}

$3.656 \pm 150$

$3.731 \pm 184$

$3.656 \pm 116$

$3.889 \pm 163$

$3.769 \pm 195$

$3.692 \pm 208$

$3.748 \pm 159$

$3.759 \pm 179$

$3.718 \pm 72$

$3.756 \pm 107$

$3.772 \pm 115$

$3.632 \pm 86$

$3.574 \pm 155$

$3.840 \pm 104$

$3.786 \pm 198$

$3.703 \pm 210$

$3.721 \pm 102$

$3.797 \pm 200$

$3.901 \pm 192$

$3.715 \pm 89$

$3.897 \pm 168$

$3.807 \pm 138$

$3.847 \pm 83$

$3.700 \pm 55$

$3.835 \pm 130$

$3.823 \pm 201$

$3.764 \pm 60$

$3.854 \pm 172$

$3.537 \pm 179$

$3.623 \pm 142$

$3.972 \pm 177$

$3.792 \pm 207$

$4.013 \pm 197$

$3.657 \pm 143$

$3.841 \pm 200$

$3.733 \pm 192$

$3.922 \pm 160$

$3.826 \pm 98$

$3.405 \pm 201$

$3.640 \pm 161$

$3.749 \pm 181$

$3.563 \pm 204$

$3.642 \pm 205$

\section{$3.180 \pm 118$}

$3.205 \pm 131$

$3.265 \pm 161$

$3.203 \pm 102$

$3.328 \pm 172$

$3.245 \pm 182$

$3.288 \pm 140$

$3.296 \pm 157$

$3.259 \pm 63$

$3.305 \pm 94$

$3.342 \pm 102$

$3.133 \pm 74$

$3.087 \pm 134$

$3.326 \pm 90$

$3.229 \pm 169$

$3.211 \pm 182$

$3.255 \pm 89$

$3.284 \pm 173$

$3.325 \pm 164$

$3.204 \pm 76$

$3.365 \pm 145$

$3.322 \pm 121$

$3.342 \pm 72$

$3.216 \pm 48$

$3.350 \pm 113$

$3.317 \pm 175$

$3.251 \pm 52$

$3.356+150$

$3.057 \pm 154$

$3.154 \pm 124$

$3.422 \pm 153$

$3.257 \pm 178$

$3.461 \pm 170$

$3.155 \pm 124$

$3.319 \pm 173$

$3.202 \pm 164$

$3.367 \pm 137$

$3.307 \pm 85$

$2.955 \pm 175$

$3.132 \pm 139$

$3.196 \pm 154$

$3.074 \pm 176$

$3.145 \pm 177$
$3.424 \pm 144$
81

82

81

85

83

81

83

84

82

83

83

82

81

85

83

82

82

82
84

85

82

86

84

84
85

83

85

84

82

82

86
79

80

85

83

88

81

83

83
84

86

84

75

82

81

79

81

precisão no processo de formulação das dietas, de modo que se possam corrigir os valores energéticos de acordo com a variação dessas composições (Albino \& Silva, 1996). Assim, a determinação de EMAn desses 45 híbridos será de grande valia na elaboração de equações de predição dos valores energéticos de diferentes amostras de milho. 
Tabela 5 - Correlações de Pearson entre as médias do coeficiente de metabolizabilidade da energia bruta (CMEB), a média dos valores da composição química e o diâmetro geométrico médio (DGM) dos híbridos de milho

Table 5 - Pearson correlations among the means of coefficient of metabolization of gross energy (CGEM) with the means of chemical composition and medium geometric diameter (MGD)

\begin{tabular}{|c|c|c|c|c|c|}
\hline $\begin{array}{l}\text { Variável } \\
\text { Variable }\end{array}$ & $\begin{array}{l}\text { Variável }^{1} \\
\text { Variable }^{1}\end{array}$ & $\begin{array}{l}\text { Observações } \\
\text { Observations }\end{array}$ & $\begin{array}{l}\text { Correlação } \\
\text { Correlation }\end{array}$ & $\mathrm{T}$ & $\begin{array}{c}\text { Significância } \\
\text { Probability }\end{array}$ \\
\hline CMEB (CGEM) & MS (DM) & 45 & $-0,1355$ & $-0,8965$ & 0,1875 \\
\hline CMEB (CGEM) & $\mathrm{EE}^{*}(E E)$ & 45 & 0,2790 & 1,9051 & 0,0317 \\
\hline CMEB (CGEM) & $\mathrm{FB}(G F)$ & 45 & $-0,0382$ & $-0,2509$ & 0,4015 \\
\hline CMEB (CGEM) & FDA (ADF) & 45 & $-0,1232$ & $-0,8140$ & 0,2101 \\
\hline CMEB (CGEM) & FDN (NDF) & 45 & $-0,2028$ & $-1,3578$ & 0,0908 \\
\hline CMEB (CGEM) & $\mathrm{Ca}$ & 45 & 0,0694 & 0,4565 & 0,3252 \\
\hline CMEB (CGEM) & $\mathrm{P}$ & 45 & 0,0334 & 0,2189 & 0,4139 \\
\hline CMEB (CGEM) & DGM (MGD) & 45 & 0,2207 & 1,4838 & 0,0726 \\
\hline
\end{tabular}

* Correlação significativa $(P<0,05)$.

* Significant correlation $(P<0.05)$.

\section{Conclusões}

A determinação da composição química do alimento, principalmente em PB, é importante para o balanceamento de rações.

A energia metabolizável aparente corrigida média dos híbridos de milho foi de $3.744 \mathrm{kcal} / \mathrm{kg}$, variando de 3.405 a $4.013 \mathrm{kcal} / \mathrm{kg}$ de MS.

\section{Literatura Citada}

ALBINO, L.F.T.; SILVA, M.A. Valores nutritivos de alimentos para aves e suínos determinados no Brasil. In: SIMPÓSIO INTERNACIONAL SOBRE EXIGÊNCIAS NUTRICIONAIS DE AVES E SUÍNOS, 1996, Viçosa, MG. Anais... Viçosa, MG: Univeridade Federal de Viçosa, 1996. p.303-318.

BATH, D.; DUBAR, J.; KING, J. et al. Byproducts and unusual feedstuffs. Feedstuffs, v.71, n.31, 1999.

EMPRESA BRASILEIRA DE PESQUISA AGROPECUÁRIA EMBRAPA. Tabela de composição química e valores energéticos de ingredientes para suínos e aves. Concórdia: EMBRAPA/CNPSA, 1991. 97p.

KATO, R.K. Valores de energia metabolizável de ingredientes para frangos de corte em diferentes idades. Lavras: Universidade Federal de Lavras. 2005. 146p. Tese (Doutorado em Zootecnia) - Universidade Federal de Lavras, 2005. Disponível em: <http://bibtede.ufla.br/tede/tde_busca/ arquivo.php?codArquivo=302) $>$ Acesso em: 05/11/06.

LIMA, G.J.M.M. Qualidade nutricional do milho: situação atual e perspectivas. In: SIMPÓSIO SOBRE MANEJO E NUTRIÇÃO DE AVES E SUÍNOS, 2000, Campinas. Anais... Campinas, 2000. p.153-174.

LIMA, G.J.M.M. Milho e subprodutos na alimentação animal. In: SIMPÓSIO SOBRE INGREDIENTES NA ALIMENTAÇÃO ANIMAL, 2001, Campinas. Anais... Campinas, 2001. p.13-32.

MATTERSON, L.D.; POTTER, L.M.; STUTZ, M.W. et al. The metabolizable energy of feed ingredients for chickens. Storrs: The University of Connecticut, Agricultural Experiment Station, 1965. 11p. (Research Report, 7).

NAGATA, A.K.; RODRIGUES, P.B.; FREITAS, R.T.F. et al. Energia metabolizável de alguns alimentos energéticos para frangos de corte, determinados por ensaios metabólicos e por equações de predição. Ciência e Agrotecnologia, v.28, n.3, p.668-677, 2004.

NATIONAL RESEARCH COUNCIL - NRC. Nutrient requirements of poultry. 9.ed. Washington: National Academy, 1994. 155p.

NUNES, R.V.; ROSTAGNO, H.S.; ALBINO, L.F.T. et al. Composição bromatológica, energia metabolizável e equações de predição da energia do grão e de subprodutos do trigo para pintos de corte. Revista Brasileira de Zootecnia, v.30, n.3, p.785-793, 2001.

RODRIGUES, P.B.; MARTINEZ, R.S.; FREITAS, R.T.F. et al. Influência do tempo de coleta e metodologia sobre a digestibilidade e o valor energético de rações para aves. Revista Brasileira de Zootecnia, v.34, n.3, p.882-889, 2005.

RODRIGUES, P.B.; ROSTAGNO, H.S.; ALBINO, L.F.T. et al. Valores energéticos do milheto, do milho, determinados com frangos de corte e galos adultos. Revista Brasileira de Zootecnia, v.30, n.6, p.1767-1778, 2001.

RODRIGUES, P.B.; ROSTAGNO, H.S.; ALBINO, L.F.T. et al. Desempenho de frangos de corte, digestibilidade de nutrientes e valores energéticos de rações formulados com vários milhos, suplementados com enzimas. Revista Brasileira de Zootecnia, v.32, n.1, p.171-182, 2003.

ROSTAGNO, H.S. Tabelas brasileiras para aves e suínos: composição de alimentos e exigências nutricionais. Viçosa, MG: Universidade Federal de Viçosa, 2000. 141p.

ROSTAGNO, H.S. Tabelas brasileiras para aves e suínos: composição de alimentos e exigências nutricionais. Viçosa, MG: Universidade Federal de Viçosa, 2005. 186p.

SANTOS, Z.A.S.; FREITAS, R.T.F.; FIALHO, E.T. et al. Valor nutricional de alimentos para suínos determinado na Universidade Federal de Lavras. Ciência e Agrotecnologia, v.29, n.1, p.232-237. 2005.

SILVA, D.J.; QUEIROZ, A.C. Análise de alimentos: métodos químicos e biológicos. 3.ed. Viçosa, MG: Universidade Federal de Viçosa, 2002. 235p.

VASCONCELLOS, C.A. Importância da adubação na qualidade do milho e sorgo. In: SIMPÓSIO SOBRE ADUBAÇÃO E QUALIDADE DOS PRODUTOS AGRÍCOLAS, 1., 1989, Ilha Solteira. Anais... Ilha Solteira: FEIS/UNESP/ANDA/POTAFÓS, 1989. p.319-330.

ZANOTTO, D.L.; BELLAVER, C. Método de determinação da granulometria de ingredientes para o uso em rações de suínos e aves. Concórdia: EMBRAPA - CNPSA, 1996. p.1-5. (Comunicado Técnico, 215). 